\title{
A self-organizing neural network for neuromuscular control
}

\author{
Praveen Shankar ${ }^{1 *}$, Sharmila Venugopal ${ }^{2}$ \\ From 24th Annual Computational Neuroscience Meeting: CNS*2015 \\ Prague, Czech Republic. 18-23 July 2015
}

Adaptive technology holds great promise for sensorimotor rehabilitation in people suffering from spinal cord injury, neuromuscular disease and stroke. With a longterm goal of developing adaptive technology for diagnosis and rehabilitation of neuromuscular dysfunction, we begin the development of a self-organizing neural network (SNN) that compensates for reduced neural drive. We suggest that the self-organizing architecture that adds or deletes nodes online to generate suitable compensatory muscle excitation (Figure 1A) is an apt mechanism to emulate the motor pool behavior of recruitment and de-recruitment of motor units during muscle force generation. Using a virtual muscle [1] resembling the human biceps brachii, we demonstrate the augmentation of neural excitation by the SNN to compensate for abnormal muscle force due to change in the number of motor units.

\section{Authors' details \\ ${ }^{1}$ Department of Mechanical and Aerospace Engineering, California State University Long Beach, CA, USA. ${ }^{2}$ Department of Integrative Biology and Physiology, University of California Los Angeles, CA, USA.}

Published: 18 December 2015

\section{Reference}

1. Cheng E, Brown I, Loeb G: Virtual muscle: a computational approach to understanding the effects of muscle properties on motor control. Journal of Neuroscience Methods 2000, 101:117-130.

doi:10.1186/1471-2202-16-S1-P277

Cite this article as: Shankar and Venugopal: A self-organizing neural network for neuromuscular control. BMC Neuroscience 2015 16(Suppl 1): P277.

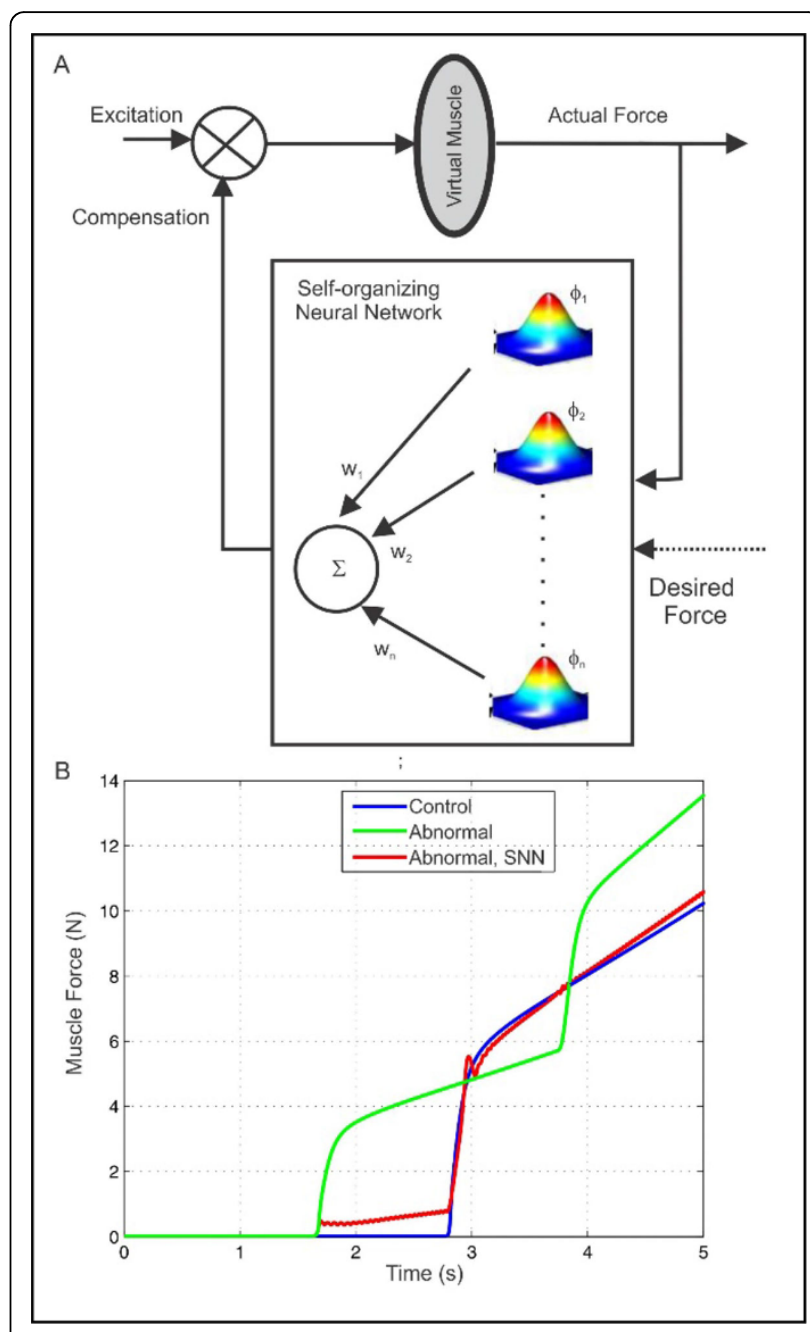

Figure $1 \mathrm{~A}$. Schematic showing the virtual muscle-SNN system; $\Phi_{1}, \Phi_{2}, . . \Phi_{n}$ are radial basis functions and $w_{1}, w_{2}, . . w_{n}$ are weights for summation. B. Simulation of normal (Slow-Fast motor unit ratio - 2:4), abnormal (Slow-Fast motor unit ratio - 3:3) muscle force and, compensation by SNN.

\footnotetext{
* Correspondence: vsharmila@ucla.edu

'Department of Mechanical and Aerospace Engineering, California State University Long Beach, CA, USA

Full list of author information is available at the end of the article
} 